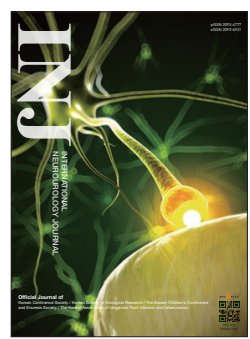

\title{
In Vitro Effects of Plasma Collected From Rats Administered Naftopidil on Whole Urinary Bladder Preparation Isolated From
}

\section{Rats}

\author{
Tsuyoshi Hattori ${ }^{1}$, Kimio Sugaya ${ }^{2}$, Saori Nishijima ${ }^{2}$, Katsumi Kadekawa ${ }^{2}$, Tomoyuki Ueda ${ }^{3}$, Hideyuki Yamamoto ${ }^{4}$ \\ ${ }^{1}$ Department of Medical Affairs, Asahi Kasei Pharma Corporation, Tokyo, Japan \\ ${ }^{2}$ Southern Knights' Laboratory, Okinawa, Japan \\ ${ }^{3}$ Institute for Animal Experiments, Faculty of Medicine, University of the Ryukyus, Okinawa, Japan \\ ${ }^{4}$ Department of Biochemistry, Graduate School of Medicine, University of the Ryukyus, Okinawa, Japan
}

\begin{abstract}
Purpose: Alpha-1-adrenoceptor blockers (e.g., naftopidil) are prescribed for the treatment of male lower urinary tract symptoms. Although the mechanism of action of naftopidil has been studied in various organs, that in the urinary bladder remains unknown. To clarify the direct effects of naftopidil on this organ, activities were assessed in the isolated rat whole urinary bladder.

Methods: A total of 30 female rats were used. In Experiment 1, bladder activity was measured during a cumulative adminis-

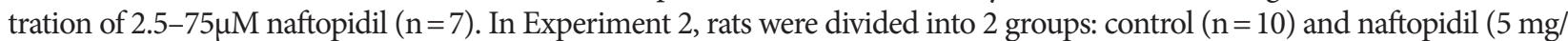
animal/day, oral gavage, once-daily for 2 weeks) $(n=13)$. After the treatment period, plasma was obtained from each rat. The urinary bladders were harvested from the control rats. Isovolumetric rhythmic bladder contractions were induced at above the threshold volume, and intravesical pressure was recorded. Control plasma was added to the organ bath; after subsequent wash-out, plasma collected from rats administered naftopidil was added. In Experiment 3, the plasma levels of monoamines and amino acids were quantified using the individual plasma prepared in the Experiment 2.

Results: Cumulative dosing with naftopidil did not change the interval between spontaneous contractions compared to the interval at baseline. After adding control plasma, the interval was shortened compared to the baseline $(\mathrm{P}=0.008)$. The plasma collected from rats administered naftopidil suppressed the shortening of the interval compared to the control plasma $(\mathrm{P}=0.041)$. Naftopidil resulted in a decrease in the level of noradrenaline $(\mathrm{P}=0.009)$ and an increase in that of glycine $(\mathrm{P}=0.014)$.

Conclusions: Although naftopidil did not directly act on the interval between spontaneous contractions of the urinary bladder, the plasma collected from rats administered naftopidil, with changing levels of monoamines and amino acids, may suppressed shortening the interval.
\end{abstract}

Keywords: Lower urinary tract symptoms; Naftopidil; Pelvic; Urinary bladder; Vascular permeability

- Grant/Fund Support: This study was supported by Asahi Kasei Pharma Corporation.

- Research Ethics: The study protocols (No. 5213, 5346, and 5599) were approved by the President of the University of the Ryukyus based on the judgment of the institutional Animal Care and Use Committee.

- Conflict of Interest: TH is an employee of Asahi Kasei Pharma Corporation. For the other authors, no potential conflict of interest relevant to this article was reported.

Corresponding author: Tsuyoshi Hattori (iD https://orcid.org/0000-0001-6425-3945 Department of Medical Affairs, Asahi Kasei Pharma Corporation, 1-1-2 Yurakucho, Chiyoda-ku, Tokyo 100-0006, Japan

E-mail: hattori.tg@om.asahi-kasei.co.jp / Tel: +81-3-6699-3718 / Fax: +81-3-6699-3655

Submitted: June 4, 2019 / Accepted after revision: September 29, 2019 (i) (7) This is an Open Access article distributed under the terms of the Creative Commons Attribution Non-Commercial License (http://creativecommons.org/licenses/by-nc/4.0/) which permits unrestricted non-commercial use, distribution, and reproduction in any medium, provided the original work is properly cited. 


\begin{abstract}
- HIGHLIGHTS
- Cumulative naftopidil did not change the interval between spontaneous contractions of isolated rat urinary bladder.

- Plasma obtained after naftopidil dosing counteracted the shortening of the interval.

- Plasma extravasation may be target for the pathogenesis of lower urinary tract symptoms.
\end{abstract}

\section{INTRODUCTION}

Lower urinary tract symptoms (LUTS) are mainly divided into 3 classes: storage symptoms, e.g., increased daytime frequency, nocturia, urgency; voiding symptoms, e.g., slow stream, intermittent stream, straining; and postmicturition symptoms, e.g., feeling of incomplete emptying, postmicturition dribble [1]. Although differences in LUTS frequency and bother are observed between men and women, in both genders the severity of LUTS increases with age and bother from LUTS increases as the severity of symptoms increases [2]. Male LUTS is often diagnosed in elderly patients, in which storage symptoms are most bothersome and interfere with daily life, decreasing the quality of life [3]. Recently, progress has been made in investigations of the pathogenesis of storage symptoms. Notably, chronic reduction of pelvic blood flow has been shown to induce increased urinary frequency in animal models and humans [4-7]. Work performed over the past decade has revealed that causes of decreased blood flow include endothelial dysfunction and oxidative stress in vascular vessels [8].

Alpha-1-adrenoceptor blockers, which are prescribed for the treatment of male LUTS, improve storage symptoms as well as voiding symptoms [9]. Naftopidil, one such a1-adrenoceptor blocker, appears to counter voiding symptoms by reducing urethral tonus [10]. This compound's activity against storage symptoms has been determined in several studies, as follows: (1) decreasing urine adenosine 5'-triphosphate levels [11], (2) regulating afferent C-fiber or spinal glycine and/or $\gamma$-amino butyric acid receptor activity $[12,13]$, and (3) potentiating blood flow in the urinary bladder [4] while also altering the level of urine nitric oxide (NO) metabolites [14]. However, it is still unknown whether naftopidil acts directly in the urinary bladder. The aim of the present study was to clarify the direct effects of naftopidil on the urinary bladder. To this end, bladder activities were assessed in the isolated rat urinary bladder.

\section{MATERIALS AND METHODS}

\section{Study Design}

The study design is summarized in Fig. 1. This study consisted of 3 experiments. In Experiment 1, the effect of cumulative naftopidil (Asahi Kasei Pharma Corp., Tokyo, Japan) exposure on the urinary bladder was assessed. In Experiment 2, the effect of the plasma prepared from naftopidil-treated and control rats (see the "Plasma Administration" section below) was examined. Urinary bladder activity, intercontraction interval, maximum contraction pressure (MCP) of the urinary bladder, and baseline intravesical pressure (BP) were measured isovolumetrically using isolated whole urinary bladders from control rats. In Experiment 3 , monoamines and amino acids were measured in the plasma from naftopidil-treated and control rats. The 3 experiments were performed separately; note, however, that while the urinary bladders used in the Experiment 1 were distinct from those used in the Experiment 2, the plasma samples assessed in Experiment 3 were the same as those generated in Experiment 2 .

\section{Animals}

Female Sprague-Dawley rats (200-240 g; Kyudo Company, Kumamoto, Japan) were used. A total of 30 rats were divided into 3 groups: the control group $(\mathrm{n}=10)$ and the naftopidil group $(\mathrm{n}=13)$ for Experiments 2 and 3; the remaining animals $(\mathrm{n}=7)$ were used for the cumulative naftopidil exposure study (Experiment 1). Rats were housed in rooms maintained at a temperature of $23^{\circ} \mathrm{C} \pm 2^{\circ} \mathrm{C}$ and relative humidity of $55 \% \pm 15 \%$ under a 12-hour on/12-hour off light/dark cycle. Animals were provided with free access to food and water throughout the studies.

\section{Drug Administration (Experiment 1)}

Naftopidil was added cumulatively to the organ bath to yield final concentrations of $2.5,7.5,25$, and $75 \mu \mathrm{M}$; bladder activities were measured at each naftopidil concentration. Serotonin (Sigma-Aldrich Co. LLC, St. Louis, MO, USA) was added into the bath to a final concentration of $1 \mathrm{mM}$ after a wash-out of the naftopidil using Ringer's solution. Finally, noradrenaline (Sankyo Co., Ltd., Tokyo, Japan) was added to final concentration of $1-2 \mathrm{mM}$ to check the activity of the bladder.

\section{Plasma Administration (Experiment 2)}

Naftopidil was suspended at $5 \mathrm{mg} / \mathrm{mL}$ in distilled water. Test 


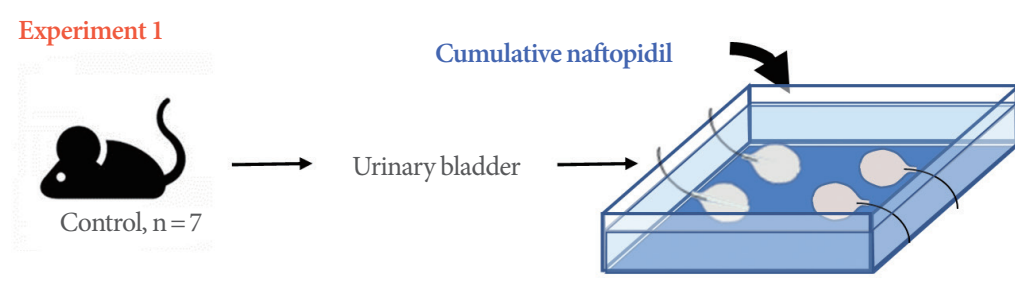

Measurement of intravesical pressure

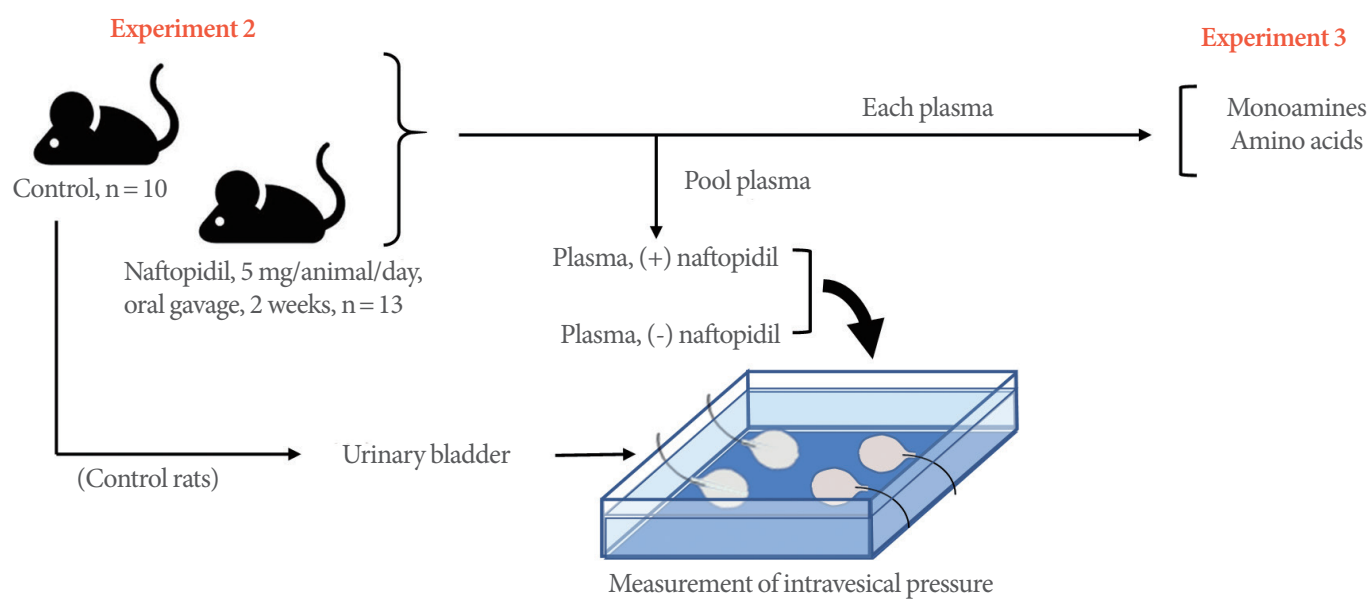

Fig. 1. Schematic of experimental design. This study consisted of 3 experiments. Details are provided in the Materials and Methods section.

article-treated rats were dosed with $5 \mathrm{mg} /$ animal/day; control rats were administered with vehicle (distilled water). All doses were administered for 2 weeks by once-daily (QD) oral gavage (PO) at $1 \mathrm{~mL} /$ animal. At 24 hours after the last dose administration, each rat was anesthetized with $2 \%$ isoflurane and whole blood was collected from inferior vena cava (as described below in "Measurement of the Bladder Activities" section) using a plasma-sampling tube (PSF; SRL, Tokyo, Japan). Plasma was isolated by centrifugation at 3,000 rpm for 10 minutes and stored on ice until used in assays. Following blood collection, the animals were euthanized and bladders were isolated from the control rats (as described below in "Measurement of the Bladder Activities" section) for use in the measurement of the bladder activities.

Aliquots of each plasma sample were stored at $-20^{\circ} \mathrm{C}$ until use for measurements of monoamines and amino acids (Experiment 3, as described below). To examine the effects of rat plasma on the bladder activity, the remaining individual plasma samples were pooled by group for tests of adding to Ringer's solution to yield a $30 \%(\mathrm{v} / \mathrm{v})$ final concentration and the bladder activities were measured. For a given bladder, the organ was sequentially exposed first to a control plasma sample, and then to
1 or 2 plasma samples prepared from naftopidil-dosed rats; each exposure was performed in the same manner, but separated by 3 wash-out intervals using undiluted Ringer's solution to eliminate the influence of the previous plasma sample.

\section{Measurements of Plasma Monoamines and Amino Acids (Experiment 3)}

The individual plasma samples were assessed for the levels of monoamines and amino acids. The monoamines (including adrenaline, noradrenaline, dopamine, and serotonin) and amino acids (including alanine, arginine, asparagine, aspartic acid, citrulline, glutamic acid, glutamine, glycine, histidine, ornithine, serine, and threonine) were quantified by high-performance liquid chromatography by SRL (Tokyo, Japan).

\section{Measurement of the Bladder Activities}

Isolation of urinary bladder and an isovolumetric recording was performed as described previously, with minor changes [15]. In brief, after inhalation anesthesia of rats with $2 \%$ isoflurane, the abdominal cavity was opened. Then, whole blood was isolated (as described above in "Plasma Administration" section) and rats were sacrificed by blood loss. Subsequently, a polyethyl- 
ene catheter (PE-50; Clay Adams, Parsippany-Troy Hills, NJ, USA) was inserted into the bladder through the urethra. The bladder neck and both ureters were ligated at once to record isovolumetric bladder contractions. The entire urinary bladder then was harvested and immediately immersed in a recording chamber filled with Ringer's solution (Otsuka Pharmaceutical Factory, Inc., Naruto, Japan) saturated with $95 \% \mathrm{O}_{2}$ and $5 \% \mathrm{CO}_{2}$ at $24^{\circ} \mathrm{C}-$ $25^{\circ} \mathrm{C}$. The reason why the Ringer's solution was set to $24^{\circ} \mathrm{C}-25^{\circ} \mathrm{C}$ for the cystometrogram is that stable contraction of frequency and amplitude was observed at this temperature range. An increase in frequency at higher temperature is induced by desynchronization of muscle activity at $20^{\circ} \mathrm{C}-38^{\circ} \mathrm{C}$, leading to the initiation of spontaneous contractions at various sites in the bladder wall [15]. Increased contractility of smooth muscle is generated at lower temperatures in the same range [15]; for example, the extent of myosin light chain phosphorylation is higher at lower temperatures than at higher temperatures [16]. The urinary bladder was fixed by pinning the urethral wall onto the resin floor of the chamber. The catheter was connected to an infusion pump (TE-331, Terumo Corporation, Tokyo, Japan) and a pressure transducer with polyethylene tubing and a three-way stopcock. The bladder was slowly filled with saline to above the threshold volume, inducing isovolumetric rhythmic bladder contractions. When the frequency and amplitude of isovolumetric contractions became stable, the contraction interval, $\mathrm{MCP}$, and $\mathrm{BP}$ were measured as control (baseline) values.

\section{Statistical Analysis}

In the measurements of bladder activity, contraction interval, $\mathrm{MCP}$, and BP were analyzed. In the experiment with plasma or serotonin application, values were compared before and after administration by using the Wilcoxon signed-rank test. Comparisons of changes by plasma or serotonin application were analyzed by the Steel-Dwass test. In the experiment with cumulative naftopidil addition, the maximum MCP values of each contraction during the measurement were averaged at each concentration of naftopidil to avoid attenuation of maximal pressure dependent on the time-course. The resulting MCP values were subjected to repeated-measurement analysis of variance with post hoc comparison to the control group using Dunnett's test. Analyses of the plasma concentrations of monoamines and amino acids were performed using the Student ttest to compare the control and naftopidil groups. All analyses were performed as 2-tailed tests using JMP ver. 14 (SAS Institute, Cary, NC, USA). $\mathrm{P}<0.05$ was considered significant. Since the analyses for monoamines and amino acids are exploratory, the issue of multiplicity was excluded.

\section{RESULTS}

\section{Bladder Activities \\ Effects of cumulative naftopidil}

The effects of cumulative naftopidil on the isolated bladders are summarized in Table 1 and a representative series is shown in Fig. 2. The MCP and BP decreased in a concentration-dependent manner, but the contraction interval did not change.

\section{Effects of rat plasma on bladder activity}

Representative effects of plasma are shown in Fig. 3. Control plasma significantly shortened the contraction interval of isolated whole urinary bladder compared to the baseline value $(\mathrm{P}=0.008)$, but control plasma did not change the MCP or the $\mathrm{BP}(\mathrm{P}=0.818$ and $\mathrm{P}=0.547$, respectively) (Fig. $4 \mathrm{~A}-\mathrm{C})$. The plasma collected from rats administered naftopidil significantly shortened the contraction interval and decreased the MCP compared to the baseline value (each $\mathrm{P}=0.016$ ); the naftopidil plasma did not similarly affect the BP $(\mathrm{P}=0.250)$ (Fig. 4D-F). Serotonin did not exhibit effects on bladder activities (Fig. 4GI). The percent reduction of contraction intervals was significantly lower with the plasma from rats administered naftopidil

Table 1. Isolated rat urinary bladder activity during cumulative naftopidil treatment

\begin{tabular}{|c|c|c|c|c|c|c|}
\hline \multirow{2}{*}{ Parameter } & \multicolumn{5}{|c|}{ Naftopidil $(\mu \mathrm{M})(\mathrm{n}=7)$} & \multirow{2}{*}{ P-value } \\
\hline & 0 & 2.5 & 7.5 & 25 & 75 & \\
\hline Contraction interval & $0.92 \pm 0.66$ & $0.85 \pm 0.08$ & $0.85 \pm 0.06$ & $0.80 \pm 0.06$ & $0.89 \pm 0.12$ & 0.844 \\
\hline Maximum contraction pressure & $37.4 \pm 2.2$ & $33.9 \pm 2.0$ & $31.2 \pm 2.1$ & $27.2 \pm 2.4^{* *}$ & $14.7 \pm 0.6^{* *}$ & $<0.001$ \\
\hline Baseline intravesical pressure & $18.4 \pm 1.3$ & $17.3 \pm 1.3$ & $15.3 \pm 1.2$ & $13.2 \pm 0.7^{\star *}$ & $12.3 \pm 0.4^{\star *}$ & 0.001 \\
\hline
\end{tabular}

Values are expressed as mean \pm standard error.

${ }^{\text {a) }}$ Repeated analysis of variance, ${ }^{* \star} \mathrm{P}<0.01$ vs. $0 \mu \mathrm{M}$ of naftopidil by Dunnett test with 2 tails. 


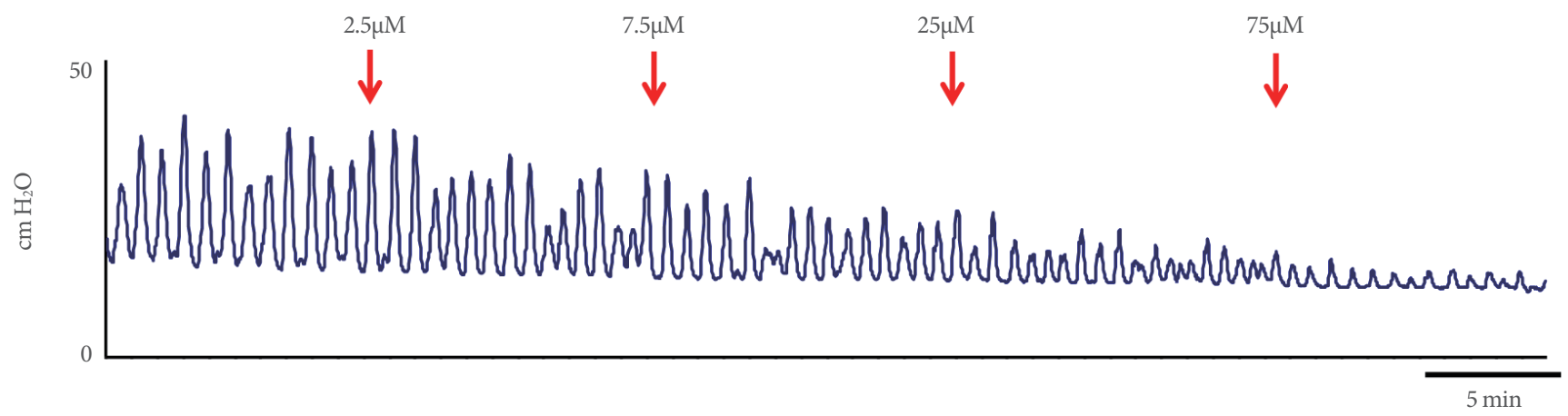

Fig. 2. Representative effects of cumulative naftopidil exposure. Arrows indicate the administration of naftopidil at each concentration. The bar indicates an interval of 5 minutes.
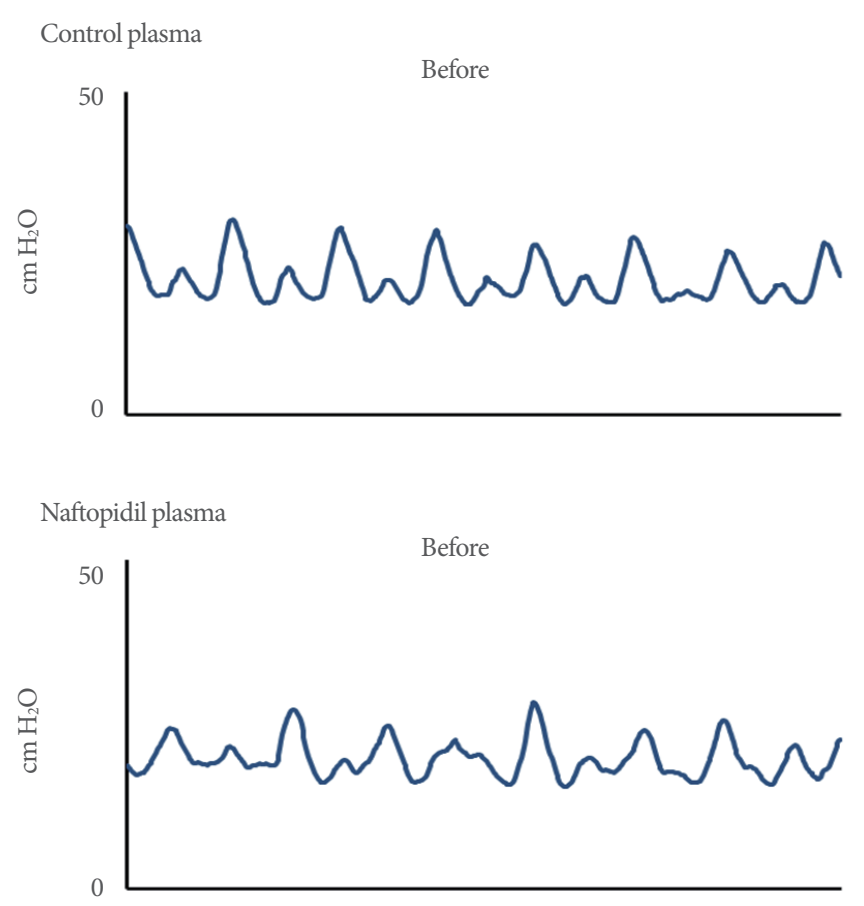

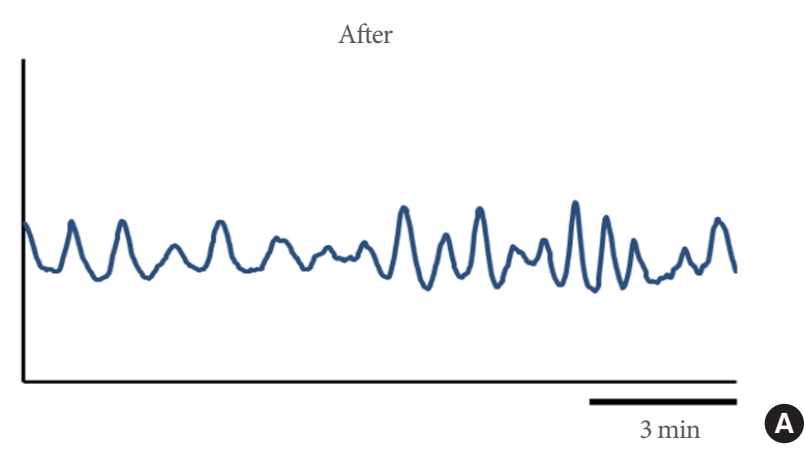

After

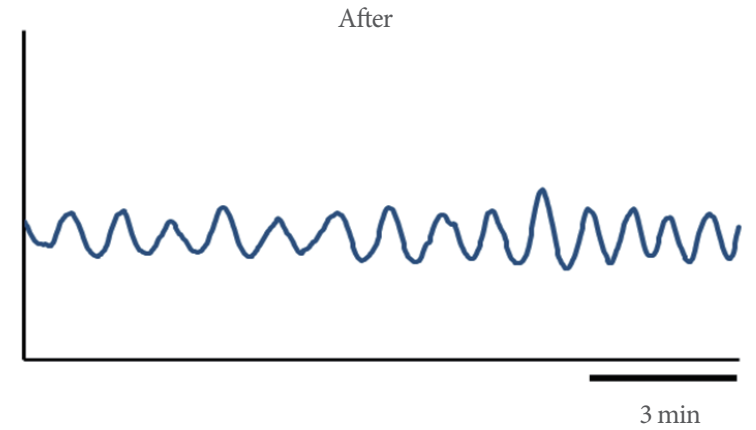

Fig. 3. Representative effects of plasma on bladder activities. (A) Cystometrograms at baseline (left panel) and after administration of control plasma (right panel). (B) Cystometrograms at baseline (left panel) and after administration of naftopidil plasma, which was collected from rats that had been administered naftopidil for 2 weeks. The bar indicates an interval of 3 minutes.

than with the plasma from the control group $(\mathrm{P}=0.041)$; in contrast, the reduction of MCP was large upon exposure to the plasma from rats administered naftopidil compared to that obtained with the plasma from the control group $(\mathrm{P}=0.011)$ (Fig. 5). Serotonin exposure did not yield significant changes in contraction interval, $\mathrm{MCP}$, and $\mathrm{BP}$, with values remaining at approximately $100 \%$ (Fig. 5). An excess concentration of noradrenaline eliminated spontaneous contractions in each bladder.

\section{Plasma Monoamines and Amino Acids}

The effects of naftopidil on the levels of plasma monoamines and amino acids are summarized in Tables 2 and 3, respectively. The level of adrenaline was significantly lowered and the levels of a subset of amino acids (asparagine, citrulline, glutamine, glycine, histidine, and threonine) were significantly elevated by naftopidil compared to control. 

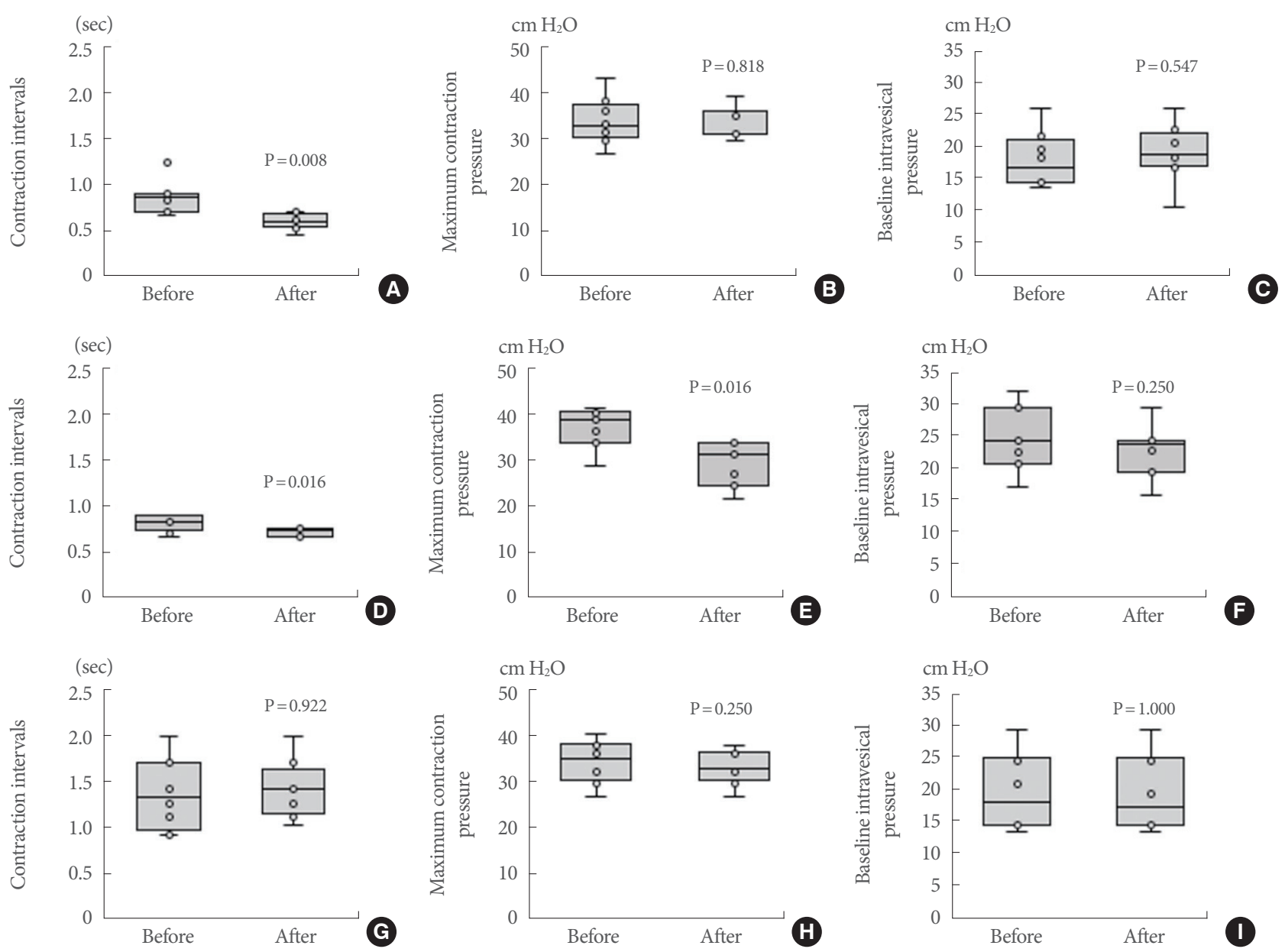

Fig. 4. Summary of cystometrogram data for the effects of plasma and serotonin. Control plasma (A-C); naftopidil plasma, which was collected from rats that had been administered naftopidil for 2 weeks (D-F); and $1 \mathrm{mM}$ serotonin (G-I) were added and the contraction interval $(\mathrm{A}, \mathrm{D}, \mathrm{G}), \mathrm{MCP}(\mathrm{B}, \mathrm{E}, \mathrm{H})$, and $\mathrm{BP}(\mathrm{C}, \mathrm{F}, \mathrm{I})$ were compared between baseline and postadministration. Data are expressed as median (25\%-75\% tile), $\mathrm{n}=7-8$, with the whiskers indicating the minimum and maximum values. P-values were determined by the Wilcoxon signed-rank test. BP, baseline pressure; MCP, maximum contraction pressure.
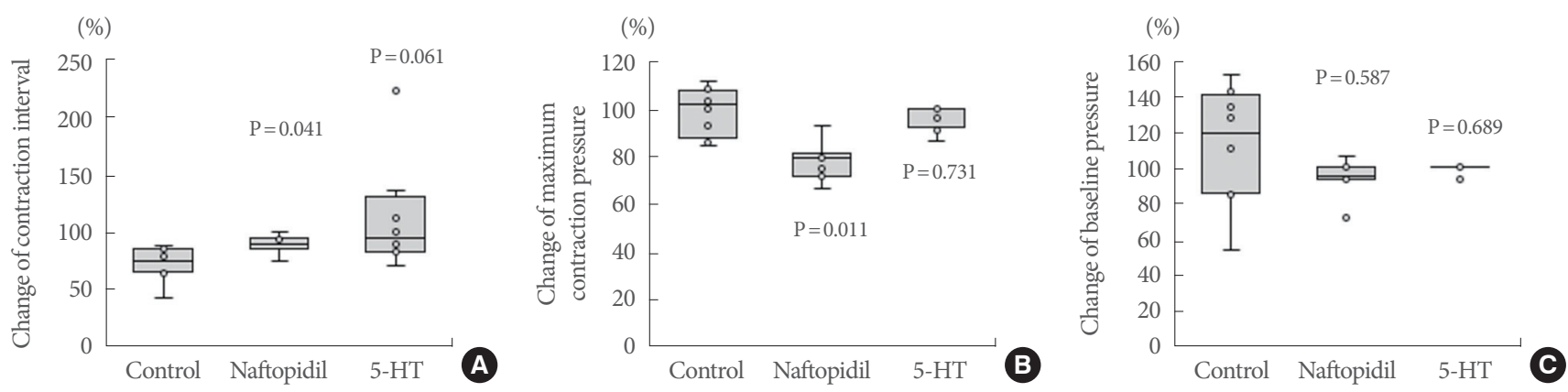

Fig. 5. Change of bladder activities and comparisons among treatments are shown for contraction interval (A), $M C P(B)$, and $B P(C)$. The ratio of values after administration to those at baseline are expressed as median $(25 \%-75 \%$ tile), $\mathrm{n}=7-8$, with the whiskers indicating the minimum and maximum values. P-values were determined by comparison to the control group using the Steel-Dwass test. BP, baseline pressure; MCP, maximum contraction pressure; 5-HT, serotonin. 
Table 2. Plasma monoamine concentrations after 2 -week administration of $5 \mathrm{mg} /$ animal naftopidil per orally (QD) in rats

\begin{tabular}{|c|c|c|c|c|c|}
\hline \multirow{2}{*}{ Parameter } & \multicolumn{2}{|c|}{ Control $(\mathrm{n}=10)$} & \multicolumn{2}{|c|}{ Naftopidil (n=13) } & \multirow{2}{*}{ P-value ${ }^{a)}$} \\
\hline & Mean \pm SE & $95 \% \mathrm{CI}$ & Mean \pm SE & $95 \% \mathrm{CI}$ & \\
\hline Adrenaline & $6,046 \pm 520$ & $4,870-7,223$ & $4,059 \pm 453$ & $3,072-5,046$ & 0.009 \\
\hline Noradrenaline & $600.8 \pm 52.3$ & $482.6-719.0$ & $667.8 \pm 49.7$ & $559.6-776.1$ & 0.368 \\
\hline Dopamine & $43.4 \pm 8.5$ & $24.1-62.7$ & $47.7 \pm 8.7$ & $28.6-66.7$ & 0.734 \\
\hline Serotonin & $381.4 \pm 61.0$ & $243.5-519.3$ & $527.7 \pm 66.3$ & $383.2-672.3$ & 0.130 \\
\hline
\end{tabular}

SE, standard error; CI, confidence interval.

Since the analyses for monoamines and amino acids are exploratory, the issue of multiplicity was excluded.

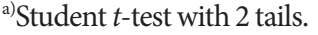

Table 3. Plasma amino acid concentrations after 2-week administration of $5 \mathrm{mg} /$ animal naftopidil per orally (QD) in rats

\begin{tabular}{|c|c|c|c|c|c|}
\hline \multirow{2}{*}{ Parameter } & \multicolumn{2}{|c|}{ Control $(\mathrm{n}=8)$} & \multicolumn{2}{|c|}{ Naftopidil $(\mathrm{n}=12)$} & \multirow{2}{*}{ P-value ${ }^{a)}$} \\
\hline & Mean \pm SE & $95 \% \mathrm{CI}$ & Mean \pm SE & $95 \% \mathrm{CI}$ & \\
\hline Alanine & $545.2 \pm 42.0$ & $445.9-644.5$ & $588.0 \pm 29.9$ & $522.3-653.7$ & 0.403 \\
\hline Arginine & $189.1 \pm 10.9$ & $163.3-214.8$ & $172.0 \pm 5.9$ & $159.1-184.9$ & 0.151 \\
\hline Asparagine & $57.1 \pm 2.9$ & $50.2-64.0$ & $64.8 \pm 2.1$ & $60.3-69.4$ & 0.039 \\
\hline Aspartic acid & $10.4 \pm 0.7$ & $8.7-12.1$ & $9.0 \pm 0.5$ & $7.9-10.1$ & 0.111 \\
\hline Citrulline & $63.8 \pm 1.8$ & $59.6-68.1$ & $70.0 \pm 2.1$ & $65.5-74.6$ & 0.049 \\
\hline Glutamic acid & $75.2 \pm 2.5$ & $69.3-81.2$ & $79.1 \pm 3.2$ & $72.0-86.2$ & 0.398 \\
\hline Glutamine & $699.3 \pm 29.4$ & $629.8-768.8$ & $837.9 \pm 19.0$ & $796.1-879.7$ & 0.001 \\
\hline Glycine & $214.9 \pm 10.6$ & $189.7-240.0$ & $256.2 \pm 10.1$ & $234.0-278.5$ & 0.014 \\
\hline Histidine & $56.1 \pm 2.3$ & $50.5-61.6$ & $63.1 \pm 2.1$ & $58.5-67.7$ & 0.040 \\
\hline Ornithine & $40.9 \pm 1.9$ & $36.4-45.3$ & $45.2 \pm 2.2$ & $40.3-50.1$ & 0.179 \\
\hline Serine & $276.3 \pm 14.8$ & $241.5-311.2$ & $307.2 \pm 8.6$ & $288.4-326.1$ & 0.068 \\
\hline Threonine & $248.0 \pm 14.6$ & $213.3-282.6$ & $288.5 \pm 11.0$ & $264.2-312.8$ & 0.037 \\
\hline
\end{tabular}

SE, standard error; CI, confidence interval.

Since the analyses for monoamines and amino acids are exploratory, the issue of multiplicity was excluded.

${ }^{\text {a) }}$ Student $t$-test with 2 tails.

\section{DISCUSSION}

The present study was intended to mimic the effect of distributed naftopidil or of naftopidil introduced into tissue of the urinary bladder by extravasating plasma, and demonstrated the following points. (1) Cumulative naftopidil did not change the contraction interval of isolated whole urinary bladder from rat; (2) plasma isolated from naftopidil-treated rats counteracted the shortening of the contraction interval; (3) naftopidil treatment yielded a decrease in the plasma level of adrenaline and increases in the plasma levels of several amino acids, including asparagine, citrulline, glutamine, glycine, histidine, and threonine.

\section{Mechanism of Action of Naftopidil in Reducing Bladder Activity}

The plasma isolated after naftopidil treatment counteracted the shortening of the contraction interval observed with the control plasma and decreased the MCP compared to baseline. These effects presumably could be attributed to either of 2 mechanisms: the presence of naftopidil itself in the plasma, and naftopidil-induced changes in the plasma levels of monoamines and amino acids. With regard to the first mechanism, the elimination half-life $\left(\mathrm{t}_{1 / 2}\right)$ of naftopidil is $12-15$ hours after a single dose of $20 \mathrm{mg} / \mathrm{kg}$ in rats [17]. In the present study, naftopidil was administered QD for 14 days as an oral dose at $5 \mathrm{mg} / \mathrm{rat} /$ day, which is equivalent to a dose of approximately $20-25 \mathrm{mg} /$ $\mathrm{kg} /$ day. Accordingly, the plasma samples, which were collected 
from rats that had been administered naftopidil approximately 24 hours earlier, still may have contained nonmetabolized naftopidil, which may have substantially suppressed the shortening of the contraction interval and decreased the MCP. In contrast, the cumulative addition of naftopidil at concentrations ranging from $2.5-75 \mu \mathrm{M}$ did not change the contraction interval of the isolated whole urinary bladder, although the compound did inhibit the MCP in a concentration-dependent manner (Table 1). Therefore, it is likely that $\alpha 1$-adrenoceptor blockade by nonmetabolized naftopidil and/or the decrease in plasma adrenaline level reduced the MCP of the isolated whole urinary bladder, but had little or no direct effect on the contraction interval. Recently, it has been reported that naftopidil reduces the level of intravesical prostaglandin $\mathrm{E}_{2}\left(\mathrm{PGE}_{2}\right)$ and attenuates the intravesical pressure of urinary bladder and the amplitude of nonvoiding contraction in rats with a partial bladder outlet obstruction [18]. Therefore, the release of $\mathrm{PGE}_{2}$ may be also associated with the increased MCP.

Serotonin did not change the bladder activities. Naftopidil has a binding affinity for the 5-HT2A and 5-HT2B receptors [19]; however, serotonin 5-HT receptors are probably excluded from the mode of action of both naftopidil itself and the plasma prepared after naftopidil treatment.

\section{Role of Changing Plasma Levels of Monoamines and Amino Acids in Reducing Bladder Activity}

Since naftopidil did not directly change the spontaneous contraction interval in the isolated urinary bladder (Table 1), the suppressive effect of the plasma, which was prepared from rats subjected to naftopidil treatment, on the interval likely reflected changes in plasma components other than naftopidil. In the present study, the plasma level of adrenaline was decreased (compared to control) following the administration of naftopidil for 2 weeks (Table 2). A $\beta 3$-adrenoceptor agonist previously was shown to prolong the contraction interval [20]. Thus, there is a discrepancy between the suppression of shortening interval observed in Experiment 2 and the decrease in the level of adrenaline seen in plasma from naftopidil-treated rats (Experiment 1). Therefore, plasma adrenaline presumably should be excluded as a candidate for the basis of the prolongation of the contraction interval by the plasma prepared after naftopidil treatment.

The 2-week administration of naftopidil elevated the plasma levels of several amino acids compared to those in control plasma (Table 3). Therefore, the suppression of shortening interval by the plasma prepared after naftopidil treatment may reflect exposure of the isolated whole urinary bladder to increased plasma levels of one or more amino acids. Notably, Nishijima et al. [21] suggested that the low level of plasma glycine may be related to anamnesis of bladder outlet obstruction or the presence of spinal cord injury. Thus, we infer that the change of the plasma level of glycine mediates bladder dysfunction when plasma is extravasated into the bladder substance and urothelium. The urinary bladder may be converted to be overactive, as seen in emerging spontaneous rhythmic contraction, so that the a1-adrenoceptor may be hyperactive. Therefore, we inferred that the reduced level of adrenaline induces decreased MCP. It has been speculated that NO could be one factor keeps the bladder relaxed during filling [22]. Increase in citrulline was presumed to result in the production of $\mathrm{NO}$ from arginine [23]. Intrathecal administration of strychnine, an antagonist of a glycine receptor, has been shown to counteract the suppression of the micturition reflex by naftopidil [12], which suggests that glycine suppressively regulates the micturition reflex. These changes in the plasma level conceivably prolong intercontraction interval, however, the further study is needed to demonstrate which factors are significant for modulating the micturition. Regarding amino acids and male LUTS, an association has been reported between the LUTS and abnormal process of amino acid metabolism [24].

\section{Possibility of Detrusor Overactivation by Plasma and Usefulness of the Isolated Whole Urinary Bladder Model}

Plasma extravasation was observed in the urinary bladder with nerve stimulation of the dorsal root at L6-S1 levels in rats [25]. The electrically induced plasma extravasation response is mediated by capsaicin-sensitive sensory fibers [26]. Therefore, afferent nerve activation suggests increased plasma extravasation following bladder activation. Animal models of pelvic venous congestion and interstitial cystitis have been reported to demonstrate high frequency or facilitation of micturition reflex; furthermore, those models show extravasation, into the tissue of the urinary bladder, of Evan's blue dye that has been administered systemically $[7,27]$. This extravasation mimics vascular hyperpermeability, which can transfer platelet and cytokines from the intravascular space to the surrounding tissues. Adenosine $5^{\prime}$-triphosphate, which is released from platelets infiltrated into the bladder tissues, likely increases detrusor contractility, as shown in a previous study [28]. Therefore, a correlation between bladder overactivity and plasma extravasation is feasi- 
ble. To evaluate this issue for contractility, the procedure using isolated whole urinary bladder is a simple and useful system that excludes the effects of autonomic innervation. In this model, bladder activity is easily assessed by measuring intravesical pressure following the addition of plasma and/or compounds. Thus, this experimental model is expected to be of use for investigating the pathogenesis of LUTS.

In conclusion, the present study indicated that naftopidil itself does not suppress directly the shortening of the contraction interval on the basis of the isolated urinary bladder. The changes of levels of monoamines and/or amino acids in the plasma isolated from rats administered naftopidil may associate bladder activity.

\section{Limitations}

This study has some limitations. The present study was performed using normal bladder. To better understand clinical practice, further experiments will be needed using bladders derived from animal models of pathological conditions. We cannot compare both effects, that is the effect of naftopidil itself added to isolated bladder and the effect of nonmetabolized naftopidil in the plasma on the activity of isolated urinary bladder, because the isolation and purification of naftopidil from the plasma is extremely challenging.

\section{AUTHOR CONTRIBUTION STATEMENT}

- Full access to all the data in the study and takes responsibility for the integrity of the data and the accuracy of the data analysis: $K S$

- Study concept and design: $K S$

- Acquisition of data: $S N$

- Analysis and interpretation of data: $S N, K S$

- Drafting of the manuscript: TH

- Critical revision of the manuscript for important intellectual content: KS

- Statistical analysis: $T H$

- Obtained funding: $K S$

- Administrative, technical, or material support: TU, KK

- Study supervision: $H Y$

\section{REFERENCES}

1. Abrams P, Cardozo L, Fall M, Griffiths D, Rosier P, Ulmsten U, et al. The standardisation of terminology of lower urinary tract func- tion: report from the Standardisation Sub-committee of the International Continence Society. Neurourol Urodyn 2002;21:167-78.

2. Apostolidis A, Kirana PS, Chiu G, Link C, Tsiouprou M, Hatzichristou D. Gender and age differences in the perception of bother and health care seeking for lower urinary tract symptoms: results from the hospitalised and outpatients' profile and expectations study. Eur Urol 2009;56:937-47.

3. Lee SW, Doo SW, Yang WJ, Song YS. Importance of relieving the most bothersome symptom for improving quality of life in male patients with lower urinary tract symptoms. Urology 2012;80:6847.

4. Majima T, Yamamoto T, Funahashi Y, Takai S, Matsukawa Y, Yoshida $\mathrm{M}$, et al. Effect of naftopidil on bladder microcirculation in a rat model of bladder outlet obstruction. Low Urin Tract Symptoms 2017;9:111-6.

5. Sugaya K, Kadekawa K, Unten Y, Nishijima S, Ashitomi K, Yamamoto $H$. Relationship of blood flow in the common iliac vein to lower urinary tract disease. J Med Ultrason (2001) 2019;46:223-9.

6. Sugaya K, Miyazato T, Koyama Y, Hatano T, Ogawa Y. Pelvic congestion syndrome caused by inferior vena cava reflux. Int J Urol 2000;7:157-9.

7. Sugaya K, Nishijima S, Kadekawa K, Noguchi K, Ashitomi K, Ueda T, et al. Pelvic venous congestion induces lower urinary tract dysfunction in rats. Biomed Res 2018;39:269-77.

8. Ismaeel A, Brumberg RS, Kirk JS, Papoutsi E, Farmer PJ, Bohannon WT, et al. Oxidative stress and arterial dysfunction in peripheral artery disease. Antioxidants (Basel) 2018 Oct 19;7(10). pii: E145. https://doi.org/10.3390/antiox7100145.

9. Gotoh M, Kamihira O, Kinukawa T, Ono Y, Ohshima S, Origasa H, et al. Comparison of tamsulosin and naftopidil for efficacy and safety in the treatment of benign prostatic hyperplasia: a randomized controlled trial. BJU Int 2005;96:581-6.

10. Takei R, Ikegaki I, Shibata K, Tsujimoto G, Asano T. Naftopidil, a novel alpha1-adrenoceptor antagonist, displays selective inhibition of canine prostatic pressure and high affinity binding to cloned human alpha1-adrenoceptors. Jpn J Pharmacol 1999;79:447-54.

11. Sugaya K, Nishijima S, Tasaki S, Kadekawa K, Miyazato M, Ogawa Y. Effects of propiverine and naftopidil on the urinary ATP level and bladder activity after bladder stimulation in rats. Neurosci Lett 2007;429:142-6.

12. Sugaya K, Nishijima S, Kadekawa K, Ashitomi K, Ueda T, Yamamoto $\mathrm{H}$. Spinal mechanism of micturition reflex inhibition by naftopidil in rats. Life Sci 2014;116:106-11.

13. Yokoyama O, Yusup A, Oyama N, Aoki Y, Tanase K, Matsuta Y, et al. Improvement of bladder storage function by alphal-blocker de- 
pends on the suppression of C-fiber afferent activity in rats. Neurourol Urodyn 2006;25:461-7.

14. Sugaya K, Nishijima S, Kadekawa K, Ashitomi K, Ueda T, Yamamoto $\mathrm{H}$. Naftopidil improves locomotor activity and urinary frequency in rats with pelvic venous congestion. Biomed Res 2016;37: 221-6.

15. Sugaya K, de Groat WC. Influence of temperature on activity of the isolated whole bladder preparation of neonatal and adult rats. Am J Physiol Regul Integr Comp Physiol 2000;278:R238-46.

16. Mitsui T, Kitazawa T, Ikebe M. Correlation between high temperature dependence of smooth muscle myosin light chain phosphatase activity and muscle relaxation rate. J Biol Chem 1994;269:5842-8.

17. Liu X, Zhang X, Huang J, Rong Y, Luo C, Guo J, et al. Enantiospecific determination of naftopidil by RRLC-MS/MS reveals stereoselective pharmacokinetics and tissue distributions in rats. J Pharm Biomed Anal 2015;112:147-54.

18. Akino H, Ito H, Nagase K, Matsuta Y, Aoki Y, Hattori T, et al. al -Blocker inhibits non-voiding contractions and decreases the level of intravesical prostaglandin E2 in rats with partial bladder outlet obstruction. Int J Urol 2019;26:924-30.

19. Sakai T, Kasahara K, Tomita K, Ikegaki I, Kuriyama H. Naftopidil inhibits 5-hydroxytryptamine-induced bladder contraction in rats. Eur J Pharmacol 2013;700:194-200.

20. Sadananda P, Drake MJ, Paton JF, Pickering AE. A functional analysis of the influence of $\beta 3$-adrenoceptors on the rat micturition cycle. J Pharmacol Exp Ther 2013;347:506-15.
21. Nishijima S, Sugaya K, Fukuda T, Miyazato M, Ashimine S, Ogawa Y. Serum amino acids as indicators of cerebrospinal neuronal activity in patients with micturition disorders. Int J Urol 2006;13:147983.

22. Andersson KE, Persson K. Nitric oxide synthase and the lower urinary tract: possible implications for physiology and pathophysiology. Scand J Urol Nephrol Suppl 1995;175:43-53.

23. Knowles RG, Moncada S. Nitric oxide synthases in mammals. Biochem J. 1994;298 (Pt 2):249-58.

24. Mitsui T, Kira S, Ihara T, Sawada N, Nakagomi H, Miyamoto T, et al. Metabolomics approach to male lower urinary tract symptoms: identification of possible biomarkers and potential targets for new treatments. J Urol 2018;199:1312-8.

25. Pintér E, Szolcsányi J. Plasma extravasation in the skin and pelvic organs evoked by antidromic stimulation of the lumbosacral dorsal roots of the rat. Neuroscience 1995;68:603-14.

26. Andrews PV, Helme RD, Thomas KL. NK-1 receptor mediation of neurogenic plasma extravasation in rat skin. Br J Pharmacol 1989; 97:1232-8.

27. Nishijima S, Sugaya K, Kadekawa K, Ashitomi K, Ueda T, Yamamoto H. High-dose tranilast administration to rats creates interstitial cystitis-like symptoms with increased vascular permeability. Life Sci 2013 26;93:897-903.

28. Saito M, Kondo A, Gotoh M, Kato K, Levin RM. Age-related changes in the response of the rat urinary bladder to neurotransmitters. Neurourol Urodyn 1993;12:191-200. 